\title{
Prenatally diagnosed submicroscopic familial aberrations at 18p11.32 without phenotypic effect
}

\author{
Malgorzata I Srebniak*, Marjan Boter, Carla MA Verboven-Peerden, Gerda AG Looye-Bruinsma, Gretel Oudesluijs, \\ Robert-Jan H Galjaard and Diane Van Opstal
}

\begin{abstract}
Background: Recent development of MLPA (Multiplex-Ligation-dependent Probe Amplification, MRC-Holland) and microarray technology allows detection of a wide range of new submicroscopic abnormalities. Publishing new cases and case reviews associated with both clinical abnormalities and a normal phenotype is of great value.

Findings/results: We report on two phenotypically normal foetuses carrying a maternally-inherited interstitial submicroscopic abnormality of chromosome 18p11.32. Both abnormalities were found with the aneuploidy MLPA kit P095 during rapid aneuploidy detection, which was offered along with conventional karyotyping. Foetus 1 and its mother have a 1,7 Mb deletion and foetus 2 and its mother have a 1,9 Mb duplication. In both cases normal babies were born. We used the HumanCytoSNP-12 array of Illumina to visualize the CNVs and map the breakpoints.
\end{abstract}

Conclusions: We suggest that a CNV at 18p11.32 $(528,050-2,337,486)$ may represent a new benign euchromatic variant.

Keywords: 18p11.32 deletion, 18p11.32 duplication, submicroscopic abnormality, normal phenotype, MLPA, SNP array

\section{Findings}

Partial 18p monosomy and 18p trisomy both refer to a chromosomal disorder resulting from the absence or duplication of a part of the short arm of chromosome 18. Clinical features of monosomy 18p include mild to moderate mental retardation, short stature, round face with short protruding philtrum, palpebral ptosis and large ears with detached pinnae [1]. Familial transmission of partial monosomy $18 \mathrm{p}$ is rare and has only been reported in a few cases [2]. 18p trisomy is a rare finding and is often associated with a quite mild and nonspecific phenotype, even when the whole arm is duplicated. Most of the patients have either an apparently normal phenotype or minor anomalies, and may or may not have mental retardation [3]. Most of the published cases have cytogenetically visible abnormalities of 18p. Recent development of MLPA (Multiplex-Ligation-dependent Probe Amplification, MRC-Holland) and microarray technology allows

\footnotetext{
* Correspondence: m.srebniak@erasmusmc.nl

Department of Clinical Genetics, Erasmus Medical Center, Rotterdam, Dr Molewaterplein 50, 3015 GE, the Netherlands
}

detection of a wide range of submicroscopic abnormalities. The application of these techniques can reveal new microdeletions/microduplications of unknown clinical relevance. Publishing new cases and case reviews associated with both clinical abnormalities and a normal phenotype is of great value.

In the present study we report on two prenatally detected cases of familial aberrations at 18p11.32 associated with a normal phenotype.

\section{Case 1}

A 38 year-old Gravida 2, Para 1 was referred for prenatal cytogenetic diagnosis because of advanced maternal age. Amniocentesis was performed at 17 weeks of gestation and rapid aneuploidy detection (RAD) by using MLPA kit P095 was offered along with conventional karyotyping. RAD on uncultured amniotic fluid cells showed a diminished signal from a terminal probe on $18 \mathrm{p}$ (the TYMS gene). The parents were informed about the MLPA finding and parental blood was sampled immediately to perform parental studies (FISH) simultaneously with prenatal
C Biomed Central 
karyotyping. The deletion was confirmed by using fluorescent in situ hybridization (FISH) with probe RP11-145B19 overlapping the TYMS ([MIM:188350]) gene. The karyotype in cultured amniotic fluid cells was 46,XX. FISH on parental chromosomes revealed that the mother carried the same deletion. The prenatal diagnosis and the parental studies (FISH) were completed within 17 days.

As the phenotype of both the mother and the foetus (the second trimester ultrasound) was normal, the parents decided to continue the pregnancy.

A healthy baby girl was born at term. She is now 2,5 years old (no dysmorphic features) and there are no indications for developmental delay or mental retardation.

To visualize the deletion and to map the breakpoints a targeted array analysis (HumanCytoSNP-12 of Illumina) was performed in a research setting according to the manufacturer instructions and analyzed by using Nexus Copy Number 5.0 (BioDiscovery software) (UCSC Mar. 2006 (NCBI36/hg18)). The array confirmed a deletion of $1,7 \mathrm{Mb}$ and the foetal karyotype was revised: 46, XX.arr 18p11.32(528,050-2,226,095)x1 mat. Figure 1 left upper panel shows the array result of chromosome 18 .

\section{Case 2}

A 28 year-old woman (Gravida 1, Para 1) was referred to our academic hospital for first trimester prenatal screening and advanced ultrasound screening after term provision of the pregnancy which showed an increased NT (Nuchal Translucency) and echo-lucent structure in thorax. At $11+4$ weeks of gestation a herniation of abdominal contents into the proximal part of the umbilical cord and a hydrothorax was seen. At $14+2$ weeks of gestation small jugular sacs were seen. The hydrothorax and herniation into the umbilical cord were resolved. Amniocentesis was performed at 16 weeks of gestation and RAD by using MLPA kit P095 was offered along with conventional karyotyping and screening for 22q11 deletion (DiGeorge Syndrome (DGS), [MIM:188400]) (MLPA kit P250). MLPA for 22q11 was normal. RAD on uncultured amniotic fluid showed an enhanced signal from a terminal probe on 18p (the TYMS gene). The parents were informed about the MLPA finding; parental blood was sampled immediately to perform parental studies simultaneously with prenatal karyotyping. The karyotype in cultured amniotic fluid cells was normal. An inter- and intrachromosomal insertion was excluded using FISH with probe RP11-145B19. MLPA on parental DNA revealed that the mother was a carrier of the same duplication. The prenatal diagnosis and the parental studies were completed within 24 days. The 1,9 Mb gain was confirmed by performing targeted array (HumanCytoSNP-12 of Illumina), analyzed by using Nexus Copy Number 5.0 (BioDiscovery software) (UCSC Mar. 2006 (NCBI36/hg18). The revised foetal karyotype was 46, XY.arr 18p11.32(431,574-2,337,486)x3 mat. The parents were also informed about the targeted array-testing, which was then reported 20 days later. In order to exclude Noonan syndrome ([MIM:163950]) (increased NT) additional tests were performed. The DNA-analysis (PTPN11 ([MIM:176876]), RAF1 ([MIM: 164760]), SOS1 ([MIM:182530]) and KRAS ([MIM: 190070])) showed no mutations. Second trimester ultrasound screening at $20+3$ gestational weeks showed no foetal abnormalities. As the phenotype of both the mother and the foetus (the second trimester ultrasound) was normal, the parents decided to continue the pregnancy. Ultrasound screening at $32+3$ also was normal. A healthy baby boy was born at term. Figure 1 right upper panel shows the array results of chromosome 18.

In 2004-2010 we have performed RAD by using MLPA kit P095 on 5764 patients (4000 [4] + 1764 samples August 2007- November 2010). These were the only two cases which showed an abnormal result with the probe specific for the TYMS gene only.

If abnormal results for the most proximal or distal chromosome regions are obtained during RAD by using MLPA or QF-PCR, they may represent clinically significant regional imbalance. These findings should be detailed in the report and further testing is recommended [5].

We report on two such cases: phenotypically normal foetuses carrying a maternally-inherited interstitial submicroscopic abnormality of chromosome 18p. Foetus 1 and its mother have a 1,7 Mb deletion and foetus 2 and its mother have a 1,9 Mb duplication. According to the UCSC human genome database the abnormal region presented here encompasses 8 genes: CETN1 ([MIM:603187]), CLUL1, C18orf56, TYMS, ENOSF1 ([MIM:607427]), YES1 ([MIM:164880]), ADCYAP1 ([MIM:102980]) and C18orf2 ([MIM:606486]). Additionally the duplication (case 2) involves also COLEC12 ([MIM:607621]). None of these genes is associated with a known genetic syndrome.

Only a few cases with a pure de novo $18 \mathrm{p}$ monosomy are described [6,7]. Most of the trisomy 18p cases are not isolated, being associated with proximal trisomy $18 \mathrm{q}$ and other segmental $18 \mathrm{q}$ imbalances. Other cases are associated with an imbalance of another chromosome resulting from the segregation of a parental translocation [3]. Correlation between genotype and phenotype when a second chromosome is involved seems to have a limited value as the second chromosome may also contribute to the phenotype [8]. There are even fewer familial cases in the literature: 8 cases of familial deletion 18p [2]. However all these patients had microscopically visible abnormality. In cases not analysed by array, it is possible that the differences in phenotype can be explained by the gene content due to different breakpoints. If the cases were not tested with a molecular technique, it might be 

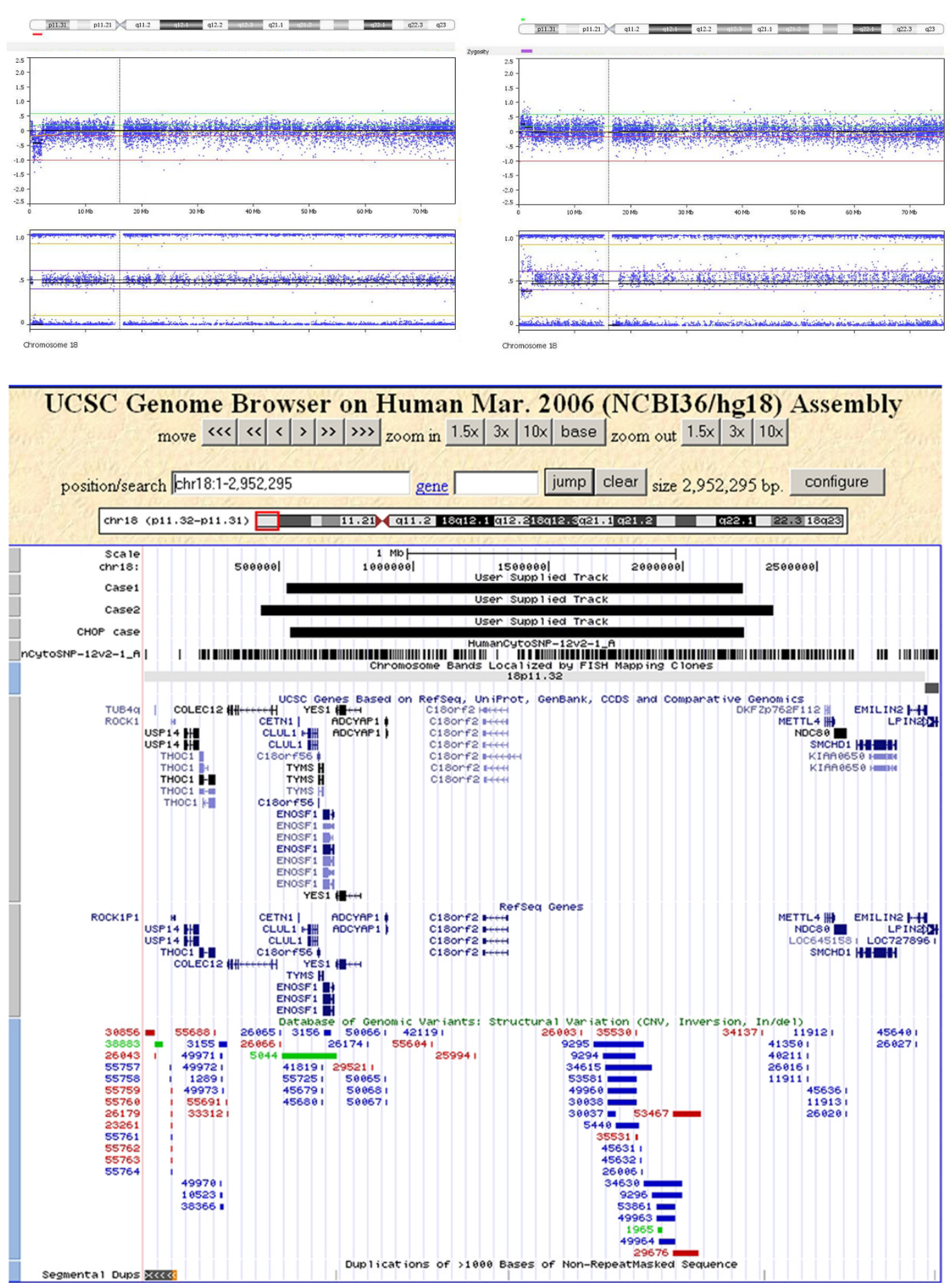

Figure 1 18p11.32 abnormalities in cases 1, 2 and CHOP patient. Left upper panel shows array results of case 1: chromosome 18 plot with $1,7 \mathrm{Mb}$ interstitial deletion of 18p11.32. Right upper panel presents array results of case 2: chromosome 18 plot with 1,9 Mb interstitial duplication of 18p11.32. Lower panel shows the results in UCSC genome browser view (Hg18), gene content of the affected region and a comparison of case 1, case 2 en CHOP case. Variations listed on DGV are visible below the reference sequences. 
even difficult to distinguish between interstitial and terminal aberrations.

Submicroscopic 18p deletions were already reported. Recently published cases with abnormal phenotype that were tested with array involve larger terminal abnormalities $[9,10]$. Terminal deletions found by using subtelomeric FISH probe or subtelomeric MLPA are also difficult to compare with our patient who has an interstitial submicroscopic aberration and normal subtelomeric regions (Figure 1) [11].

In case of such subtle abnormalities we search our own databases, Toronto Database of Genomic Variants (DGV) (http://projects.tcag.ca/variation/), Children's Hospital of Philadelphia (CHOP) database (http://cnv.chop.edu) [12], Decipher (http://decipher.sanger.ac.uk), ISCA database (https://www.iscaconsortium.org/) and literature looking for similar cases in a normal and abnormal population. We have found one normal individual with a deletion of comparable breakpoints and size and a normal phenotype in $\mathrm{CHOP}$ database. We believe that the child from CHOP database with a 1,68 Mb deletion (chr18:541,688$2,230,220)$ together with our unaffected cases (2 children and their mothers) represents the fifth healthy individual with an interstitial abnormality of the same region on 18p. Figure 1 lower panel shows the three CNVs: case 1 (deletion 18p11.32), case 2 (duplication 18p11.32), the CHOP European individual (deletion 18p11.32) and the gene content of the affected region.

There are also a few comparable cases in Decipher database, which overlap with our patients. Patients 257164 and 253425 respectively have a $0,07 \mathrm{Mb}$ gain and 0,51 Mb gain inherited from a normal parent, which supports our theory that a CNV within $18 \mathrm{p} 11.32(528,050-2,337,486)$ is most probably a benign variation. However there are also two other patients with an abnormal phenotype one with a 1,29 Mb gain of unknown inheritance (Decipher 253424) and one with a de novo 1,18 Mb loss (Decipher 257509). Whether 18p CNV is the only genomic aberration in these patients is unclear. Although 18p is not yet known as imprinted region the parental origin of the CNV might also explain the variability of the phenotype. In our cases both abnormalities were inherited from a normal mother.

Because there is only one case of a patient with an abnormal phenotype and a de novo $\mathrm{CNV}$ and in total 7 normal individuals (4 current cases, $1 \mathrm{CHOP}$ and 2 Decipher normal parents) carrying a CNV within $18 \mathrm{p} 11.32$ $(528,050-2,337,486)$ we suggest that a CNV in this region may represent a new benign euchromatic variant.

\section{Consent}

Patients undergoing prenatal diagnosis at our medical university are informed that we may investigate (publish) their medical data as long as all data remained anonymised. Each patient had the opportunity to object to their inclusion within the published data. No objections were made to this publication.

\section{Authors' contributions}

MIS coordinated the study, wrote the paper and studied the literature. MB performed the MLPA and microarray analyses. CAMVP and GAGLB performed cytogenetic analyses. GO did the genetic counselling of the parents, coordinated by R-JHG. MIS, R-JHG and DVO were responsible for the final (molecular) cytogenetic diagnoses and reports. All authors read and approved the manuscript.

\section{Competing interests}

The authors declare that they have no competing interests.

Received: 14 July 2011 Accepted: 2 December 2011

Published: 2 December 2011

\section{References}

1. Turleau C: Monosomy 18p. Orphanet J Rare Dis 2008, 3:4.

2. Misceo D, Orstavik KH, Lybaek H, Sandvig I, Ormerod E, Houge G, Frengen $\mathrm{E}$ : Inheritance of a terminal $7.1 \mathrm{Mb} 18 \mathrm{p}$ deletion flanked by a $2.3 \mathrm{Mb}$ duplication from a physically normal mother. Am J Med Genet A 2009, 149A:2877-2881.

3. Marical H, Le Bris MJ, Douet-Guilbert N, Parent P, Descourt JP, Morel F, De Braekeleer M: 18p trisomy: a case of direct 18p duplication characterized by molecular cytogenetic analysis. Am J Med Genet A 2007, 143A:2192-2195.

4. Van Opstal D, Boter M, de Jong D, van den Berg C, Bruggenwirth HT, Wildschut HI, de Klein A, Galjaard RJ: Rapid aneuploidy detection with multiplex ligation-dependent probe amplification: a prospective study of 4000 amniotic fluid samples. Eur J Hum Genet 2009, 17:112-121.

5. Professional Guidelines for clinical cytogenetics and clinical molecular genetics: QF-PCR for the Diagnosis of Aneuploidy Best Practise Guidelines. [http://www.cytogenetics.org.uk/].

6. Taine L, Goizet C, Wen ZQ, Chateil JF, Battin J, Saura R, Lacombe D: 18p monosomy with midline defects and a de novo satellite identified by FISH. Ann Genet 1997, 40:158-163.

7. Tonk V, Krishna J: Case report: denovo inherited $18 p$ deletion in a mother-fetus pair with extremely variable expression, confirmed by fluorescence in situ hybridization (FISH) analysis. Eur J Obstet Gynecol Reprod Biol 1997, 73:193-196.

8. Brenk CH, Prott EC, Trost D, Hoischen A, Walldorf C, Radlwimmer B, Wieczorek D, Propping P, Gillessen-Kaesbach G, Weber RG, Engels $H$ : Towards mapping phenotypical traits in 18p-syndrome by array-based comparative genomic hybridisation and fluorescent in situ hybridisation. Eur J Hum Genet 2007, 15:35-44.

9. Abu-Amero KK, Hellani A, Salih MA, Alorainy IA, Zidan G, Kern KC, Sicotte NL, Bosley TM: Optic disk and white matter abnormalities in a patient with a de novo 18p partial monosomy. Ophthalmic Genet 2010, 31:147-154.

10. Zavala J, Ramirez M, Medina R, Heard P, Carter E, Crandall A, Hale D, Cody J, Escamilla M: Psychiatric syndromes in individuals with chromosome 18 abnormalities. Am J Med Genet B Neuropsychiatr Genet 2010, 153B:837-845.

11. Babovic-Vuksanovic D, Jenkins SC, Ensenauer R, Newman DC, Jalal SM: Subtelomeric deletion of $18 p$ in an adult with paranoid schizophrenia and mental retardation. Am J Med Genet A 2004, 124A:318-322.

12. Shaikh TH, Gai X, Perin JC, Glessner JT, Xie H, Murphy K, O'Hara R, Casalunovo T, Conlin LK, D'Arcy $M$, et al: High-resolution mapping and analysis of copy number variations in the human genome: a data resource for clinical and research applications. Genome Res 2009, 19:1682-1690.

doi:10.1186/1755-8166-4-27

Cite this article as: Srebniak et al:: Prenatally diagnosed submicroscopic familial aberrations at 18p11.32 without phenotypic effect. Molecular Cytogenetics 2011 4:27. 